\title{
Epigenetic regulation mediated by miRNA in the susceptibility and pathogenesis of rheumatoid arthritis
}

Shicheng Guo ${ }^{1,2 \# \$}$, Cen Chang ${ }^{3,4 \#, ~ L i n g x i a ~ X u ̈ 3,4 \#, ~ R u n r u n ~ Z h a n g ~}{ }^{4}$, Yehua Jin ${ }^{4}$, Momiao Xiong ${ }^{5}$, Dongyi $\mathrm{He}^{3,4,6 \$}$

1, Department of Medical Genetics, School of Medicine and Public Health, University of Wisconsin-Madison, Madison, WI, 53726, USA.

2, Center for Precision Medicine Research, Marshfield Clinic Research Institute, Marshfield, WI, 54449, USA

3, Shanghai University of Traditional Chinese Medicine, Shanghai, 200052, China

4, Department of Rheumatology, Shanghai Guanghua Hospital, Shanghai University of Traditional Chinese Medicine, Shanghai 200052, China.

5, Department of Biostatistics and Data Science, The University of Texas Health Science Center at Houston School of Public Health 1200 Herman Pressler, Houston, TX 77030

6, Arthritis Institute of Integrated Traditional and Western medicine, Shanghai Chinese Medicine Research Institute, Shanghai 200052, China.

*SG, CC and LX contribute equally to the study.

${ }^{*}$ Correspondence:

Shicheng Guo, Ph.D.

Department of Medical Genetics

School of Medicine and Public Health

University of Wisconsin-Madison, Madison

Tel: 281-685-5882

Email: Shicheng.Guo@wisc.edu

Dongyi He, M.D., Ph.D.

Department of Rheumatology

Shanghai Guanghua Hospital, Shanghai University of Traditional Chinese Medicine

Shanghai, China

Tel : 158-0030-0800

Email: hedongyi1967@shutcm.edu.cn

\section{Contribution:}

micro-RNA (miRNA) has been demonstrated to play important roles in the transcriptome regulation and disease development including cancer and autoimmune disease such as rheumatoid arthritis. However, a comprehensive role of miRNAs in rheumatoid arthritis (RA) including immune system differentiation and interaction with terminal cells like T cells, fibroblast-like synoviocytes (FLS), osteoblast and osteoclast still unclear. In this review, we have provided a thorough summary on the roles of miRNAs in the susceptibility, pathogenesis, diagnosis, therapeutic intervention and prognosis. We summarized the blood and cell-free miRNA biomarkers which provided novel opportunity to work together with rheumatoid factors (RF), anti-CCP to provide accurate diagnosis and prognosis especially for seronegative patients. Finally, miRNAs were showed as promising biomarker to indicate the DMRDS and immunotherapy efficiency, drug response and resistance. What's more, autotherapeutic effect of miRNA intervention provided promising to develop miRNA based rheumatoid arthritis drugs. Overall, current evidence supports miRNAs as the interesting targets to better understand the pathogenetic mechanism and therapeutic intervention of rheumatoid arthritis. 


\begin{abstract}
micro-RNA (miRNA) has been demonstrated to play important roles in the transcriptome regulation and disease development including cancer and autoimmune disease such as rheumatoid arthritis. However, a comprehensive map on how the mRNAs regulate transcripts, pathways, immune system differentiation and interaction with terminal cells like T cells, fibroblast-like synoviocytes (FLS), osteoblast and osteoclast still unknown. In this review, we have provided a thorough summary on the roles of miRNAs in the susceptibility, pathogenesis, diagnosis, therapeutic intervention and prognosis. Numerous miRNAs were found abnormally expressed in rheumatoid arthritis relevant cells and regulated the target genes and pathways like $N F-K B$, Fas-FasL, JAK-STAT, IRE1-RIDD, mTOR pathway. In addition, miRNA act as gene expression regulators affect the $T$ cell differentiate to different cell types including Th17 and T-reg cells which provide promising gene therapy target to regulate immune systems in rheumatoid arthritis. We also summarized interesting diagnosis and prognosis roles of blood and cell-free based miRNAs which provided novel opportunity to work together with rheumatoid factors (RF), anti-CCP to provide accurate diagnosis and prognosis especially for seronegative patients. Furthermore, functional genetic variants in miR-499 and miR-146a explained part of missing susceptibility of rheumatoid arthritis. Finally, miRNAs were showed as promising biomarker to indicate the DMRDS and immunotherapy efficiency, drug response and resistance. What's more, autotherapeutic effect of miRNA intervention provided promising to develop miRNA based rheumatoid arthritis drugs. Overall, current evidence supports miRNAs as the interesting targets to better understand the pathogenetic mechanism and therapeutic intervention of rheumatoid arthritis.
\end{abstract}

Key words: rheumatoid arthritis, miRNA, susceptibility, pathogenesis, Epigenetics

Running title: Epigenetic regulation mediated by miRNA in Rheumatoid arthritis

\title{
Introduction:
}

Rheumatoid arthritis (RA) is an autoimmune disease with manifestation of chronic joint inflammation and structural damage and accompanied by extra-articular manifestations such as rheumatoid nodules, interstitial pneumonia, vasculitis, and systemic complications. Besides, it is usually progressive and insidious(1). However, currently, the underlying cause of RA's pathogenesis, disease activity, severity and difference in treatment effect are not fully understood. Under current rheumatoid arthritis therapy strategy and treatment frame, early accurate diagnosis and effective and personalized treatment and precision medicine have become the urgent problems for RA which required further and comprehensive understanding of rheumatoid arthritis including from the angles of both genetics(2) (HLA and non- $H L A$ variants) and epigenetics (DNA methylation(3, 4), microRNA(5, 6), IncRNA(7, 8) and histone modifications(9)).

miRNA is a small endogenous non-coding single-stranded RNA family with a length of about 22 nucleotides and is involved in the regulation of post-transcriptional gene expression. In recent years, more and more studies have found that miRNAs play an important role in a variety of cancer(10-14) and autoimmune diseases including RA, systemic lupus erythematosus $(\operatorname{SLE})(15,16)$, Sjogren's syndrome (SS)(17) and systemic sclerosis (SSc)(18). In this review, we systemically summarized the advance of the miRNA research in rheumatoid arthritis especially the relationship between the genetic variants, expression variations and susceptibility, pathogenesis of rheumatoid arthritis in different inflammation-related cells, inflammatory cytokines and inflammatory signaling pathways (Figure 1). 
Genome-wide association studies have identified $>100$ genetic factors for RA. However, these reported genetic variants only explain $<40 \%$ overall heritability of RA. Majority of the heritability is still missing which require to be identified with more studies with different approaches and populations. Association study to miRNA locus provided an opportunity to identify RA associated functional or causal variants within different population Chinese(19, 20), Egyptians(21-23), Polish(24), Mexican(25), and Iranians(26). rs3746444 (20q11.22, $A>G$ ) in miR-499 and intron of $M Y H 7 B$ was demonstrated to be significantly linked to RA risk, disease activity, and methotrexate toxicity(27) in which The AA genotype had higher disease activity and methotrexate toxicity compared with AG/GG genotypes(28). In addition, gene expression and genetic polymorphisms of miR-146a and miR-499 showed diagnostic potentials for rheumatoid arthritis(23). rs2910164 in miR-146a was identified to be associated with RA susceptibility in the Egyptian population, in which C allele was protective $(23,28)$. rs3027898 in IRAK1 which is the target gene of miR-146a was demonstrated associated RA in Greece population(29). However, follow-up studies showed inconsistent result in Poland(24), Mexico(25) and China(19, 20, 30, 31) population. SNPs located in other miRNAs were also tested in some studies however the association is not quite significant. For example, miR-196a-2 (rs11614913C/T) and miR-499 (rs3746444A/G) were showed do not have significant association with RA in Mexico population(25). miR-146a (rs2910164)(20,31) and miR-499 (rs3746444)(20, 30) were found do not have significant association with RA in Chinese. In our recent study, we found meta-analysis could identify more significant SNPs with large samples size and we found the interaction between HLA alleles and miRNA SNPs (rs5997893 in miR-3928 and rs4947332 in HLA-DRB1) should be paid further attention to explain unmet susceptibility(32).

\section{Gene and signaling pathways regulated by miRNA in the development of rheumatoid arthritis.}

The cells involved in the pathogenesis of RA include CD4+ $\mathrm{T}$ cells such as Th1, T-reg and Th17 cells, FLS, osteoclasts, and macrophages. The current research mainly focusing to identify miRNAs mediated transcriptional regulation to $F A F 1(33), \operatorname{TNF}-\alpha(34,35), \operatorname{STAT1}(36), \operatorname{STAT3}(37), \operatorname{TLR} 4(38)$ and $m T O R(39,40)$. Therefore, the miRNAs receive the regulatory roles to inflammation, immune response, proliferation, differentiation and environment involvement within synovial joints mediated by above mentioned genes and related pathways including Fas-FasL pathway(33) and $N F-k B$ pathway(34, 41, 42). In this section, we summarized the miRNA mediated regulation roles in the main RA associated cell entities including $T$ cells, FLS, osteoclasts to explain the importance of miRNAs in the pathogenesis of RA.

\section{miRNAs mediated innate and adaptive immune cell differentiation in rheumatoid arthritis}

The balance of T-reg/Th17 cells plays an important role in RA. IL-17 produced by Th17, which up-regulates Receptor Activator of Nuclear Factor-kB Ligand (RANKL), expression on synovial fibroblasts and induces innate immune cells to produce inflammatory cytokines such as $T N F-\alpha, I L-6$ and $I L-1(43)$. The regulatory roles of miR-146a were widely studied in $T$ cells and the evidence showed miR-146a was significantly increased in $\mathrm{CD}^{+} \mathrm{T}$ cells, PBMC and Jurkat $\mathrm{T}$ cells, promoting $\mathrm{T}$ cell differentiation and inhibiting apoptosis (33, 44). Interestingly, the expression of miR-146a reduced in T-reg cells during high activity of RA, by targeting STAT1, resulting the proinflammatory phenotype of T-reg cells(36). Besides that, the high level of $m i R-99 b-5 p(40)$, miR-361-5p(45) and miR-17(46) participated in enhancing $\mathrm{T}$ cell proliferation and differentiation, anti-apoptosis. miR-99b-5p in peripheral blood mononuclear cells (PBMCs) was found to decrease the expression of mTOR and RASSF4 genes to inhibit T cell apoptosis, promoted T cell proliferation and inflammatory response(40). Upregulation of miR-17 in RA exosomes could inhibit the differentiation of T-reg by inhibiting the expression of TGFB2(46). miR-21 decreased in peripheral blood circulating PBMCs(47), while increased in $\mathrm{V}$ Y $9 \mathrm{~V} \delta 2 \mathrm{~T}$ cells $(48)$ in RA patients, which associated with the imbalance of Th17/T-reg cells. miR-120 negative regulation of HIF-1 affects the dynamic equilibrium of Th17/T-reg cells. 
Unfortunately, there was no association was found for miR-120 in RA(49). In addition to inducing $T$ cell differentiation, DNA methylation induced by miRNA plays an important role in the pathogenesis of RA. miR126 inhibited the methylation of promoter region in CD70 and CD11a to promote the expression of CD11a and CD70(50). The binding of miR-6089 and IncRNA-HIX003209 promoted the expression of TLR4 and exacerbates inflammation through the $T L R 4 / N F-k B$ pathway in macrophages(51). miR-30a could increase inflammation by aggravating autophagy and reducing apoptosis(52). Over-expression of miR-192-5p improves inflammatory response in RA by targeting up-regulation of Ras-related C3 botulinum toxin substrate 2 (RAC2)(53). Increasing the expression of $m i R-20$ and miR-211 would subsequently down-regulate ATF2, thereby reducing inflammation in $\mathrm{RA}(54)$. The decreased expression of miR-671, miR-96 in PBMC of RA patients may be related to the expression of $m T O R(39)$ and endoplasmic reticulum stress induced by inositolrequiring enzyme 1 alpha (IRE1) / endoplasmic reticulum stress $(R I D D)$ pathway(55). miR-29b enhances anti-apoptotic ability of PBMCs by inhibiting HMG-box transcription factor 1(HBP1) signaling(56). miR-198, miR-4647 and miR-7167-5p were related to $T$ cell signaling, apoptosis, immune response(57). There were numerous miRNA expression related to the T-reg subpopulation, miR-21 and miR-155 are related to the memory phenotype, while miR-92a is related to the naive phenotype(58). miR-223 is highly expressed in naive CD4 ${ }^{+} \mathrm{T}$ cell, but hardly expressed in Th17(59). Overexpression of miR-361-5p in early RA was associated with $\mathrm{T}$ cell activation and inflammatory response(45). These special expressions may be involved in the pathogenesis of RA, which deserve further research. Overall, miRNAs work together with DNA methylation and other non-coding RNAs ( $\mathrm{ncRNA}$ ) regulate the innate and adaptive immune cell differentiation, apoptosis, and then involved in the inflammation and autoimmune response in rheumatoid arthritis.

\section{miRNAs mediated fibroblast-like synoviocytes and osteocyte differentiation in rheumatoid arthritis}

Synovial fibroblasts are key regulators of inflammation and bone destruction in rheumatoid arthritis. In addition to produce $R A N K L$, fibroblast-like synoviocytes in RA (C) also activates osteoclast differentiation by producing inflammatory cytokines, chemokines and matrix metalloproteinases (MMPs)(60). Large number evidence showed down-regulation of $\operatorname{miR}-22(61)$, $m i R-29 c-3 p(62), \operatorname{miR}-124 a(63), \operatorname{miR}-4701-5 p(8)$, and upregultion of $m i R-143(64), m i R-145(64)$ and $m i R-191(65)$ increased the proliferation, migration and invasion of RA-FLS. In contrast, down-regulation of $m i R-132-3 p(62)$, miR-29a(37) and up-regulation of miR-31-5p(62) and miR-124a(66) inhibited the proliferation, migration and invasion of RA-FLS(62). miR-199a-3p(67), miR$449 a(68)$, miR-506(38), and miR-126(35) are decreased in RA and targeted $R B 1$, high-mobility group box protein 1 (HMGB1), TLR4, and IL-23R, and finally inhibited RA-FLS proliferation and induces apoptosis. The proliferation and invasion of RA-FLS is related to MMP(69). In RA-FLS, the up-regulation of miR-145-5p(41), $\operatorname{miR}-18 a(34), \operatorname{miR}-155(70,71), \operatorname{miR}-203(42)$, and the down-regulation of $m i R-27 a(72)$ were participated in MMP expression through NF-KB pathway(34, 41, 42), TLR4(63), and Follistain like-1 (FSTL1) (72). miR-625 was down-regulated in RA-FLS and negatively regulated CTSC, KLF8, EBF3. miR-551b was up-regulated in RA-FLS of RA and it down-regulated ITGBL1. CTSC, KLF8, EBF3 and ITGBL1 which are related to RA-FLS phenotype differentiation(73). In RA, bone loss is mainly due to overabsorption of bone by osteoclasts and weakened osteoblast bone formation(74). Overexpression of miR-221-3p inhibited osteoblast differentiation(75). miR-218 overexpression inhibited the Roundabout 1(ROBO1)/Dickkopf-1 (DKK1) axis to promote osteogenic differentiation of RA-FLS(76).

\section{Blood and serum-based miRNAs provided novel opportunity to the diagnosis of rheumatoid arthritis}

The expression of $m i R-371 b, m i R-483, m i R-642 b$ is significantly up-regulated while $m i R-25, m i R-378 d$ are down-regulated in PBMC that eventually developed from early undifferentiated arthritis (EUA) into RA(77). Meanwhile, miR-22(78), miR-361-5p(45) and miR-223-3p(45) are significantly up-regulated in high-risk or CCP positive populations. Therefore, miR-642b-5p, miR-483-3p, miR-371b-5p, miR-25-3p, miR-378d, miR22, $m i R-361-5 p$ and $m i R-223-3 p$ can be used as biomarkers for early diagnosis of RA. miR-103a-3p are 
significant up-regulated in autoantibody-positive, asymptomatic first-degree relatives (FDR) and RA patients, indicates it can be a potential biomarker for predicting imminent disease in individuals at risk for developing RA(79). In addition, higher levels of miR-143-3p, miR-145-5p, miR-99b-5p are found in the plasma of early RA patients with bone erosion indicating the ability to be used for bone erosion surveillance in RA patients. Furthermore, miR-99b-5p was demonstrated to be an independent predictor of bone erosion progression in early $\mathrm{RA}(80)$. In addition to play role in early recognition of RA, the expression of some miRNAs aids to diagnose RA with higher accuracy(81). The expression of miR-146a and miR-155 are significantly increased in RA PBMC, and there was a similar expression trend in whole blood(82). The expressions of miR-24 and miR-125a are significantly up-regulated in the serum of RA patients regardless of CCP status(83). The combination of miR-24-3p, miR-26a-5p and miR-125a-5p showed better meet the diagnostic criteria of RA patients, However, they are not related to disease activities(84). miR-122-3p, miR-3925-3p, miR-342-3p and miR-4764-5p are differentially expressed not only in healthy individuals and RA patients, but also in RA patients and OA, SLE, and Graves patients(85). What's more, miR-4634, miR-181d, miR-3926, miR-3926, miR-9-5p, miR-219-2-3p6, miR-221, miR-222, miR-532, miR-106a and miR-987 are also expressed differentially in the serum of RA patients which can be used as RA-specific diagnostic markers $(85,86)$.

The levels of $m i R-146 a(87), \operatorname{miR}-22-3 p(88), \operatorname{miR}-5571-3 p(89) \quad$ and $m i R-135 b-5 p(89)$ in the serum of the RA group are significantly higher than those in the healthy group and the OA group. In addition, the expression of miR-451 in T cells of RA group is significantly increased, which is positively correlated with the levels of DAS28, ESR and serum IL-6(58). miR-146a is positively correlated with ESR and DAS28; miR-5571-3p is positively correlated with ESR and CRP and $m i R-135 b-5 p$ is positively correlated with CRP. Therefore, miR146a, miR-5571-3p, miR-135b-5p and miR-451 can be used as markers of disease activity in RA patients. In addition, an increase in serum miR-194-5p levels is associated with disease recurrence(90). The Serum expression of $m i R-23 b$ which positively correlated with ESR, CRP and DAS28 are significantly up-regulated after treatment, indicating that $m i R-23 b$ is a dual marker of disease activity and prognosis(91). miR-96-5p, miR-134-5p, miR-140-3p, miR-627-5p are not only diagnostic markers for RA, but also indicates the disease activity(92). In summary, the changes of miRNA in serum concentrations provided a promising opportunity for the early diagnosis, disease activity indicating and prediction of outcomes to RA.

\section{Role of miRNAs in pharmacogenetics and therapeutic outcome and response prediction}

The common and widely used anti-rheumatic drugs include cDMARD (Methotrexate, Sulfasalazine and Hydroxychloroquine), bDMARD (TNF inhibitors, Rituximab and Tocilizumab) and tsDMARD (Tofatinib, Barretinib and Flgotinib). Several studies have explored the relationship between serum miRNA levels and the response of DMARD. Evidence shows high serum levels of miR-10 in RA patients is found related to the well response to $\mathrm{MTX}(93)$. After 3 months ADA/MTX combined treatment, serum level of miR-27a-3p is significantly decreased and the clinical symptoms of RA have remission(94). Serum level of miR-5196 is significantly decreased in RA and AS patients after anti-TNF- $\alpha$ therapy and indicates a lower DAS28 scores(95). Meanwhile, serum level of miR-146a are showed decreased in RA patients who responded well to anti-TNF therapy and showed interesting response prediction ability to anti-TNF- $\alpha$ therapy $(24,96,97)$ together with CRP. In contrast, serum level of miR-23 and miR-223 are increased in RA patients who response well to anti-TNF-a/DMARD combination therapy, while negative correlated to the response to antiTNF drugs(96). High serum levels of $m i R-125 b$ can be used to an indicator to well clinical response to Rituximab therapy(98). The expression of $m i R-432-5 p$ decreased significantly in RA patients who are effective to tofacitinib therapy, but the expression of $m i R-432-5 p$ increased in RA relapse patients(90). The treatment of Rituximab can increase the levels of $m i R-16-5 p$ and miR-23a-3p in peripheral blood with $\mathrm{RA}(99)$. miR-425-5p, miR-21-5p and miR-212-3p are significantly decreased in RA patients treated with glucocorticoids, but no clinical response studies have been conducted(100). In addition to DMARD treatment, alternative and complementary medicine preparations and mesenchymal stem cell (MSC) treatments are 
also used in the clinical treatment of RA. miR-550b-2-5p, miR-4797-5p, miR-6509-5p, miR-378g, miR-4720$5 p$, miR-374b-5p and miR-185-3p are showed differently expressed between individuals who have well and worse response to Tripterysium Glycosides (TG) treatment $(101,102)$. miR-26b-5p, miR-487b-3p, and miR495-3p are up-regulated significantly in the responders with adipose-derived mesenchymal stem cell (eASCs) treatment(103). Furthermore, Geniposide treatment can increase the levels of miR-124a in FLS. However, the relevance with clinical response studies do mot conducted(104).

The auto therapeutic effect of miRNAs has been demonstrated in mouse models of RASF and autoimmune arthritis. miR-506 mimics was showed to reduce the proliferation of RA-FLS and the production of proinflammatory cytokines. Meanwhile it can promote the apoptosis of RA-FLS(38). miR-449a mimics can inhibit proliferation, migration and IL-6 production by regulating HMGB1 and YY1 expression in RA-FLS(68). In the autoimmune arthritis mouse model, the vein injection of miR-708-5p mimic showed improved pathological changes in CIA rat models including inhibit inflammatory cell infiltration, synovial hyperplasia and cartilage destruction(105). miR-126 agonist can inhibit the expression of IL-23R, TNF- $\alpha$ and IFN- $y$ in the FLS of CIA rat model(35). MSC-derived miR-124a exosomes inhibit the proliferation and migration and promote the apoptosis of fibroblast-like synovial cell lines(66). In addition, exo-miR-150 has been shown to inhibit RA FLS proliferation and angiogenesis and reduce RA joint destruction by targeting MMP14 and VEGF in rat RASF and CIA rat models(106). In conclusion, miRNA was demonstrated very important roles in the treatment of rheumatoid arthritis not only as the outcome biomarkers but also could be taken as novel drug target to decrease the severity of the patients of rheumatoid arthritis.

\section{Challenge and opportunity of the current miRNA research in rheumatoid arthritis.}

miRNAs play multiple roles in the development of RA from susceptibility and pathogenesis. As an important biomarker, blood and serum miRNA have been developed to different biomarkers for the early diagnosis and drug response prediction. Furthermore, miRNA has been proposed to be autotherapeutic approach and as the novel drug target for the treatment of rheumatoid arthritis. Genetic variants in specific miRNAs increase or decrease the risk and disease activity of RA in different ethnicity, and they are associated with methotrexate toxicity and other treatment response. In addition, the changes in miRNAs in various cells are related to the pathogenesis and pathological changes of RA, such as the proliferation and differentiation of immune cells, the proliferation and apoptosis of synovial cells, the synovial inflammation and cartilage destruction. Current research has received significant progress to develop miRNAs as the biomarkers for the diagnosis, prognosis, disease activity and the response to therapeutic drugs with RA, providing a direction for early diagnosis and accurate treatment of RA to receive better treatment efficiency and precision medicine. A variety of miRNAs have been shown to act as therapeutic targets in RA-FL and CIA rat models. miRNA also showed promising ability to identify subtypes of RA, for example, the expression levels of miR-7 and miR-214-5p are significantly increased in the serum of RA-ILD patients(107) while miR-9-5p targeted REST/miR-132 pathway to protects Schwann cells from inflammatory damage in RA-induced peripheral neuropathy(108). Although we have achieved exciting progress to investigate the multiple roles of miRNA in RA, more relevant studies should be implemented to understand and transfer current knowledge into the clinical application and solve the current inconsistent result among different studies with different method or populations. For example, Studies of miR-99, miR-143 and miR-197 as the landmark miRNAs for prediction to response to anti-TNF- $\alpha$ therapy have failed to yield results consistent with previous studies(109). Finally, we expect miRNA-based baseline rheumatoid arthritis polygenetic risk score model especially work together with HLA. Meanwhile, we miRNA based early diagnosis, prognosis and drug response prediction models could be applied in the future clinical application and miRNA based autotherapeutic treatment could show more promising result and more miRNA-based drug target could be identified in the further clinical research.

\section{Author contributions}


SG, DH conceived of content. SG, CC and LX drafted the review, which was edited by RZ, YJ and MX.

\section{Competing interests}

No potential conflicts of interest was disclosed for all the authors

\section{Funding}

This work was funded by the National Natural Science Funds of China (81774114), Shanghai Chinese Medicine Development Office, Shanghai Chinese and Western Medicine Clinical Pilot Project (ZY(2018 2020)-FWTX-1010), Shanghai Chinese Medicine Development Office, Shanghai Traditional Chinese Medicine Specialty Alliance Project (ZY(2018-2020)-FWTX-4017), National Administration of Traditional Chinese Medicine, Regional Chinese Medicine (Specialist) Diagnosis and Treatment Center Construction Project-Rheumatology.

\section{Reference}

1. Ibanez-Cabellos JS, Seco-Cervera M, Osca-Verdegal R, Pallardo FV, Garcia-Gimenez JL. Epigenetic Regulation in the Pathogenesis of Sjogren Syndrome and Rheumatoid Arthritis. Front Genet (2019) 10:1104. Epub 2019/12/05. doi: 10.3389/fgene.2019.01104. PubMed PMID: 31798626; PubMed Central PMCID: PMCPMC6863924.

2. Okada Y, Wu D, Trynka G, Raj T, Terao C, Ikari K, et al. Genetics of rheumatoid arthritis contributes to biology and drug discovery. Nature (2014) 506(7488):376-81. Epub 2014/01/07. doi: 10.1038/nature12873. PubMed PMID: 24390342; PubMed Central PMCID: PMCPMC3944098.

3. Guo S, Zhu Q, Jiang T, Wang R, Shen Y, Zhu X, et al. Genome-wide DNA methylation patterns in CD4+ T cells from Chinese Han patients with rheumatoid arthritis. Mod Rheumatol (2017) 27(3):441-7. Epub 2016/09/03. doi: 10.1080/14397595.2016.1218595. PubMed PMID: 27585642.

4. Chen S, Pu W, Guo S, Jin L, He D, Wang J. Genome-Wide DNA Methylation Profiles Reveal Common Epigenetic Patterns of Interferon-Related Genes in Multiple Autoimmune Diseases. Front Genet (2019) 10:223. Epub 2019/04/27. doi: 10.3389/fgene.2019.00223. PubMed PMID: 31024609; PubMed Central PMCID: PMCPMC6459983.

5. Mizoguchi F, Kohsaka H. [Role of microRNA in rheumatoid arthritis]. Nihon Rinsho Meneki Gakkai Kaishi (2012) 35(1):69-74. Epub 2012/03/01. doi: 10.2177/jsci.35.69. PubMed PMID: 22374446.

6. Furer V, Greenberg JD, Attur M, Abramson SB, Pillinger MH. The role of microRNA in rheumatoid arthritis and other autoimmune diseases. Clin Immunol (2010) 136(1):1-15. Epub 2010/03/13. doi: 10.1016/j.clim.2010.02.005. PubMed PMID: 20223711.

7. Guo S, Liu J, Jiang T, Lee D, Wang R, Zhou X, et al. (5R)-5-Hydroxytriptolide (LLDT-8) induces substantial epigenetic mediated immune response network changes in fibroblast-like synoviocytes from rheumatoid arthritis patients. Sci Rep (2019) 9(1):11155. Epub 2019/08/03. doi: 10.1038/s41598-019-47411-1. PubMed PMID: 31371761; PubMed Central PMCID: PMCPMC6671973.

8. Bi X, Guo XH, Mo BY, Wang ML, Luo XQ, Chen YX, et al. LncRNA PICSAR promotes cell proliferation, migration and invasion of fibroblast-like synoviocytes by sponging miRNA-4701-5p in rheumatoid arthritis. EBioMedicine (2019) 50:408-20. Epub 2019/12/04. doi: 10.1016/j.ebiom.2019.11.024. PubMed PMID: 31791845; PubMed Central PMCID: PMCPMC6921299. 9. Angiolilli C, Kabala PA, Grabiec AM, Van Baarsen IM, Ferguson BS, Garcia S, et al. Histone deacetylase 3 regulates the inflammatory gene expression programme of rheumatoid arthritis fibroblast-like synoviocytes. Ann Rheum Dis (2017) 76(1):27785. Epub 2016/07/28. doi: 10.1136/annrheumdis-2015-209064. PubMed PMID: 27457515; PubMed Central PMCID: PMCPMC5264225. 
10. Shen F, Chen J, Guo S, Zhou Y, Zheng Y, Yang Y, et al. Genetic variants in miR-196a2 and miR-499 are associated with susceptibility to esophageal squamous cell carcinoma in Chinese Han population. Tumour Biol (2016) 37(4):4777-84. Epub 2015/11/01. doi: 10.1007/s13277-015-4268-3. PubMed PMID: 26518769.

11. Fan L, Chen L, Ni X, Guo S, Zhou Y, Wang C, et al. Genetic variant of miR-4293 rs12220909 is associated with susceptibility to non-small cell lung cancer in a Chinese Han population. PLoS One (2017) 12(4):e0175666. Epub 2017/04/15. doi: 10.1371/journal.pone.0175666. PubMed PMID: 28410417; PubMed Central PMCID: PMCPMC5391943.

12. He Y, Cui Y, Wang W, Gu J, Guo S, Ma K, et al. Hypomethylation of the hsa-miR-191 locus causes high expression of hsamir-191 and promotes the epithelial-to-mesenchymal transition in hepatocellular carcinoma. Neoplasia (2011) 13(9):841-53. Epub 2011/10/05. doi: 10.1593/neo.11698. PubMed PMID: 21969817; PubMed Central PMCID: PMCPMC3182276.

13. Lin S, Pan L, Guo S, Wu J, Jin L, Wang JC, et al. Prognostic role of microRNA-181a/b in hematological malignancies: a meta-analysis. PLoS One (2013) 8(3):e59532. Epub 2013/03/28. doi: 10.1371/journal.pone.0059532. PubMed PMID: 23533632; PubMed Central PMCID: PMCPMC3606212.

14. Zhang P, Wang J, Lu T, Wang X, Zheng Y, Guo S, et al. miR-449b rs10061133 and miR-4293 rs12220909 polymorphisms are associated with decreased esophageal squamous cell carcinoma in a Chinese population. Tumour Biol (2015) 36(11):8789-95. Epub 2015/06/10. doi: 10.1007/s13277-015-3422-2. PubMed PMID: 26055141.

15. Xie L, Xu J. Role of MiR-98 and Its Underlying Mechanisms in Systemic Lupus Erythematosus. J Rheumatol (2018) 45(10):1397-405. Epub 2018/06/17. doi: 10.3899/jrheum.171290. PubMed PMID: 29907673.

16. Senousy MA, Helmy HS, Fathy N, Shaker OG, Ayeldeen GM. Association of MTMR3 rs12537 at miR-181a binding site with rheumatoid arthritis and systemic lupus erythematosus risk in Egyptian patients. Sci Rep (2019) 9(1):12299. Epub 2019/08/25. doi: 10.1038/s41598-019-48770-5. PubMed PMID: 31444373; PubMed Central PMCID: PMCPMC6707250.

17. Jang SI, Tandon M, Teos L, Zheng C, Warner BM, Alevizos I. Dual function of miR-1248 links interferon induction and calcium signaling defects in Sjogren's syndrome. EBioMedicine (2019) 48:526-38. Epub 2019/10/11. doi: 10.1016/j.ebiom.2019.09.010. PubMed PMID: 31597594; PubMed Central PMCID: PMCPMC6838412.

18. Iwamoto N, Vettori S, Maurer B, Brock M, Pachera E, Jungel A, et al. Downregulation of miR-193b in systemic sclerosis regulates the proliferative vasculopathy by urokinase-type plasminogen activator expression. Ann Rheum Dis (2016) 75(1):30310. Epub 2014/11/12. doi: 10.1136/annrheumdis-2014-205326. PubMed PMID: 25384965.

19. Yang B, Chen J, Li Y, Zhang J, Li D, Huang Z, et al. Association of polymorphisms in pre-miRNA with inflammatory biomarkers in rheumatoid arthritis in the Chinese Han population. Hum Immunol (2012) 73(1):101-6. Epub 2011/10/25. doi: 10.1016/j.humimm.2011.10.005. PubMed PMID: 22019503.

20. Yang B, Zhang JL, Shi YY, Li DD, Chen J, Huang ZC, et al. Association study of single nucleotide polymorphisms in premiRNA and rheumatoid arthritis in a Han Chinese population. Mol Biol Rep (2011) 38(8):4913-9. Epub 2010/12/25. doi: 10.1007/s11033-010-0633-x. PubMed PMID: 21181275.

21. El-Shal AS, Aly NM, Galil SM, Moustafa MA, Kandel WA. Association of microRNAs genes polymorphisms with rheumatoid arthritis in Egyptian female patients. Joint Bone Spine (2013) 80(6):626-31. Epub 2013/06/05. doi: 10.1016/j.jbspin.2013.03.005. PubMed PMID: 23731641.

22. Shaker OG, El Boghdady NA, El Sayed AE. Association of MiRNA-146a, MiRNA-499, IRAK1 and PADI4 Polymorphisms with Rheumatoid Arthritis in Egyptian Population. Cell Physiol Biochem (2018) 46(6):2239-49. Epub 2018/05/08. doi: 10.1159/000489592. PubMed PMID: 29734142.

23. Ayeldeen G, Nassar Y, Ahmed H, Shaker O, Gheita T. Possible use of miRNAs-146a and -499 expression and their polymorphisms as diagnostic markers for rheumatoid arthritis. Mol Cell Biochem (2018) 449(1-2):145-56. Epub 2018/04/28. doi: 10.1007/s11010-018-3351-7. PubMed PMID: 29700729.

24. Bogunia-Kubik K, Wysoczanska B, Piatek D, Iwaszko M, Ciechomska M, Swierkot J. Significance of Polymorphism and Expression of miR-146a and NFkB1 Genetic Variants in Patients with Rheumatoid Arthritis. Arch Immunol Ther Exp (Warsz) (2016) 64(Suppl 1):131-6. Epub 2017/01/14. doi: 10.1007/s00005-016-0443-5. PubMed PMID: 28083614; PubMed Central PMCID: PMCPMC5334424.

25. Aleman-Avila I, Jimenez-Morales M, Beltran-Ramirez O, Barbosa-Cobos RE, Jimenez-Morales S, Sanchez-Munoz F, et al. Functional polymorphisms in pre-miR146a and pre-miR499 are associated with systemic lupus erythematosus but not with rheumatoid arthritis or Graves' disease in Mexican patients. Oncotarget (2017) 8(54):91876-86. Epub 2017/12/02. doi: 10.18632/oncotarget.19621. PubMed PMID: 29190882; PubMed Central PMCID: PMCPMC5696148. 
26. Hashemi M, Eskandari-Nasab E, Zakeri Z, Atabaki M, Bahari G, Jahantigh M, et al. Association of pre-miRNA-146a rs2910164 and premiRNA-499 rs3746444 polymorphisms and susceptibility to rheumatoid arthritis. Mol Med Rep (2013) 7(1):287-91. Epub 2012/11/10. doi: 10.3892/mmr.2012.1176. PubMed PMID: 23138379.

27. Toraih EA, Ismail NM, Toraih AA, Hussein MH, Fawzy MS. Precursor miR-499a Variant but not miR-196a2 is Associated with Rheumatoid Arthritis Susceptibility in an Egyptian Population. Mol Diagn Ther (2016) 20(3):279-95. Epub 2016/03/24. doi: 10.1007/s40291-016-0194-3. PubMed PMID: 27002721.

28. Fattah SA, Ghattas MH, Saleh SM, Abo-Elmatty DM. Pre-micro RNA-499 Gene Polymorphism rs3746444 T/C is Associated with Susceptibility to Rheumatoid Arthritis in Egyptian Population. Indian J Clin Biochem (2018) 33(1):96-101. Epub 2018/01/27. doi: 10.1007/s12291-017-0652-7. PubMed PMID: 29371777; PubMed Central PMCID: PMCPMC5766468.

29. Chatzikyriakidou A, Voulgari PV, Georgiou I, Drosos AA. A polymorphism in the 3'-UTR of interleukin-1 receptorassociated kinase (IRAK1), a target gene of miR-146a, is associated with rheumatoid arthritis susceptibility. Joint Bone Spine (2010) 77(5):411-3. Epub 2010/09/28. doi: 10.1016/j.jbspin.2010.05.013. PubMed PMID: 20870441.

30. Yang XK, Li P, Zhang C, Leng RX, Li S, Liu J, et al. Association between IRAK1 rs3027898 and miRNA-499 rs3746444 polymorphisms and rheumatoid arthritis : A case control study and meta-analysis. Z Rheumatol (2017) 76(7):622-9. Epub 2016/09/02. doi: 10.1007/s00393-016-0169-0. PubMed PMID: 27581002.

31. Zhou X, Zhu J, Zhang H, Zhou G, Huang Y, Liu R. Is the microRNA-146a (rs2910164) polymorphism associated with rheumatoid arthritis? Association of microRNA-146a (rs2910164) polymorphism and rheumatoid arthritis could depend on gender. Joint Bone Spine (2015) 82(3):166-71. Epub 2015/01/30. doi: 10.1016/j.jbspin.2014.12.009. PubMed PMID: 25630616. 32. Shicheng Guo YJ, Jieru Zhou, Qi Zhu, Ting Jiang, Yanqin Bian, Runrun Zhang, Steven J Schrodi, Dongyi He. MicroRNA variants and HLA-miRNA interactions are novel rheumatoid arthritis susceptibility factors. Annals of the Rheumatic Diseases (Under Review) (2020).

33. Li J, Wan Y, Guo Q, Zou L, Zhang J, Fang Y, et al. Altered microRNA expression profile with miR-146a upregulation in CD4+ T cells from patients with rheumatoid arthritis. Arthritis Res Ther (2010) 12(3):R81. Epub 2010/05/13. doi:

10.1186/ar3006. PubMed PMID: 20459811; PubMed Central PMCID: PMCPMC2911863.

34. Trenkmann M, Brock M, Gay RE, Michel BA, Gay S, Huber LC. Tumor necrosis factor alpha-induced microRNA-18a activates rheumatoid arthritis synovial fibroblasts through a feedback loop in NF-kappaB signaling. Arthritis Rheum (2013) 65(4):916-27. Epub 2013/01/03. doi: 10.1002/art.37834. PubMed PMID: 23280137.

35. Gao J, Kong R, Zhou X, Ji L, Zhang J, Zhao D. MiRNA-126 expression inhibits IL-23R mediated TNF-alpha or IFN-gamma production in fibroblast-like synoviocytes in a mice model of collagen-induced rheumatoid arthritis. Apoptosis (2018) 23(11-

12):607-15. Epub 2018/09/01. doi: 10.1007/s 10495-018-1474-7. PubMed PMID: 30167920; PubMed Central PMCID: PMCPMC6208910.

36. Zhou Q, Haupt S, Kreuzer JT, Hammitzsch A, Proft F, Neumann C, et al. Decreased expression of miR-146a and miR-155 contributes to an abnormal Treg phenotype in patients with rheumatoid arthritis. Ann Rheum Dis (2015) 74(6):1265-74. Epub 2014/02/25. doi: 10.1136/annrheumdis-2013-204377. PubMed PMID: 24562503.

37. Liu J, Fei D, Xing J, Du J. MicroRNA-29a inhibits proliferation and induces apoptosis in rheumatoid arthritis fibroblast-like synoviocytes by repressing STAT3. Biomed Pharmacother (2017) 96:173-81. Epub 2017/10/11. doi:

10.1016/j.biopha.2017.09.120. PubMed PMID: 28987940.

38. Li D, Zhou Q, Hu G, Wang G. MiRNA-506 inhibits rheumatoid arthritis fibroblast-like synoviocytes proliferation and induces apoptosis by targetting TLR4. Biosci Rep (2019) 39(5). Epub 2019/04/13. doi: 10.1042/BSR20182500. PubMed PMID: 30975731; PubMed Central PMCID: PMCPMC6505192.

39. Tang X, Wang J, Xia X, Tian J, Rui K, Xu H, et al. Elevated expression of ciRS-7 in peripheral blood mononuclear cells from rheumatoid arthritis patients. Diagn Pathol (2019) 14(1):11. Epub 2019/02/04. doi: 10.1186/s13000-019-0783-7. PubMed PMID: 30711014; PubMed Central PMCID: PMCPMC6359828.

40. Zhu X, Wu L, Mo X, Xia W, Guo Y, Wang M, et al. Identification of PBMC-expressed miRNAs for rheumatoid arthritis. Epigenetics (2020) 15(4):386-97. Epub 2019/10/08. doi: 10.1080/15592294.2019.1676613. PubMed PMID: 31588838.

41. Wang X, Tang K, Wang Y, Chen Y, Yang M, Gu C, et al. Elevated microRNA1455p increases matrix metalloproteinase9 by activating the nuclear factorkappaB pathway in rheumatoid arthritis. Mol Med Rep (2019) 20(3):2703-11. Epub 2019/07/20. doi: 10.3892/mmr.2019.10499. PubMed PMID: 31322192; PubMed Central PMCID: PMCPMC6691224. 
42. Stanczyk J, Ospelt C, Karouzakis E, Filer A, Raza K, Kolling C, et al. Altered expression of microRNA-203 in rheumatoid arthritis synovial fibroblasts and its role in fibroblast activation. Arthritis Rheum (2011) 63(2):373-81. Epub 2011/02/01. doi: 10.1002/art.30115. PubMed PMID: 21279994; PubMed Central PMCID: PMCPMC3116142.

43. van Hamburg JP, Tas SW. Molecular mechanisms underpinning T helper 17 cell heterogeneity and functions in rheumatoid arthritis. Journal of autoimmunity (2018) 87:69-81. doi: 10.1016/j.jaut.2017.12.006. PubMed PMID: 29254845.

44. Niimoto T, Nakasa T, Ishikawa M, Okuhara A, Izumi B, Deie M, et al. MicroRNA-146a expresses in interleukin-17 producing T cells in rheumatoid arthritis patients. BMC Musculoskelet Disord (2010) 11:209. Epub 2010/09/16. doi: 10.1186/1471-2474-11-209. PubMed PMID: 20840794; PubMed Central PMCID: PMCPMC2950393.

45. Romo-Garcia MF, Bastian Y, Zapata-Zuniga M, Macias-Segura N, Castillo-Ortiz JD, Lara-Ramirez EE, et al. Identification of putative miRNA biomarkers in early rheumatoid arthritis by genome-wide microarray profiling: A pilot study. Gene (2019) 720:144081. Epub 2019/09/02. doi: 10.1016/j.gene.2019.144081. PubMed PMID: 31473322.

46. Wang L, Wang C, Jia X, Yu J. Circulating Exosomal miR-17 Inhibits the Induction of Regulatory T Cells via Suppressing TGFBR II Expression in Rheumatoid Arthritis. Cell Physiol Biochem (2018) 50(5):1754-63. Epub 2018/11/02. doi:

10.1159/000494793. PubMed PMID: 30384383.

47. Dong L, Wang X, Tan J, Li H, Qian W, Chen J, et al. Decreased expression of microRNA-21 correlates with the imbalance of Th17 and Treg cells in patients with rheumatoid arthritis. J Cell Mol Med (2014) 18(11):2213-24. Epub 2014/08/29. doi: 10.1111/jcmm.12353. PubMed PMID: 25164131; PubMed Central PMCID: PMCPMC4224555.

48. Guggino G, Orlando V, Saieva L, Ruscitti P, Cipriani P, La Manna MP, et al. Downregulation of miRNA17-92 cluster marks Vgamma9Vdelta2 T cells from patients with rheumatoid arthritis. Arthritis Res Ther (2018) 20(1):236. Epub 2018/10/24. doi: 10.1186/s13075-018-1740-7. PubMed PMID: 30348222; PubMed Central PMCID: PMCPMC6235230.

49. Huang Q, Chen SS, Li J, Tao SS, Wang M, Leng RX, et al. miR-210 expression in PBMCs from patients with systemic lupus erythematosus and rheumatoid arthritis. Ir J Med Sci (2018) 187(1):243-9. Epub 2017/06/01. doi: 10.1007/s11845-0171634-8. PubMed PMID: 28560518.

50. Yang G, Wu D, Zeng G, Jiang O, Yuan P, Huang S, et al. Correlation between miR-126 expression and DNA hypomethylation of CD4+ T cells in rheumatoid arthritis patients. Int J Clin Exp Pathol (2015) 8(8):8929-36. Epub 2015/10/16. PubMed PMID: 26464634; PubMed Central PMCID: PMCPMC4583866.

51. Yan S, Wang P, Wang J, Yang J, Lu H, Jin C, et al. Long Non-coding RNA HIX003209 Promotes Inflammation by Sponging miR-6089 via TLR4/NF-kappaB Signaling Pathway in Rheumatoid Arthritis. Front Immunol (2019) 10:2218. Epub 2019/10/18. doi: 10.3389/fimmu.2019.02218. PubMed PMID: 31620132; PubMed Central PMCID: PMCPMC6759987.

52. Xu K, Xu P, Yao JF, Zhang YG, Hou WK, Lu SM. Reduced apoptosis correlates with enhanced autophagy in synovial tissues of rheumatoid arthritis. Inflamm Res (2013) 62(2):229-37. Epub 2012/11/28. doi: 10.1007/s00011-012-0572-1. PubMed PMID: 23178792.

53. Zheng J, Zhu L, Iok In I, Chen Y, Jia N, Zhu W. Bone marrow-derived mesenchymal stem cells-secreted exosomal microRNA-192-5p delays inflammatory response in rheumatoid arthritis. International immunopharmacology (2020) 78:105985. Epub 2019/11/30. doi: 10.1016/j.intimp.2019.105985. PubMed PMID: 31776092.

54. Li B, Li N, Zhang L, Li K, Xie Y, Xue M, et al. Hsa_circ_0001859 Regulates ATF2 Expression by Functioning as an MiR204/211 Sponge in Human Rheumatoid Arthritis. J Immunol Res (2018) 2018:9412387. Epub 2018/03/27. doi:

10.1155/2018/9412387. PubMed PMID: 29577053; PubMed Central PMCID: PMCPMC5822876.

55. Ahmadiany M, Alavi-Samani M, Hashemi Z, Moosavi MA, Rahmati M. The Increased RNase Activity of IRE1alpha in PBMCs from Patients with Rheumatoid Arthritis. Adv Pharm Bull (2019) 9(3):505-9. Epub 2019/10/09. doi:

10.15171/apb.2019.060. PubMed PMID: 31592115; PubMed Central PMCID: PMCPMC6773928.

56. Ren B, Liu J, Wu K, Zhang J, Lv Y, Wang S, et al. TNF-alpha-elicited miR-29b potentiates resistance to apoptosis in peripheral blood monocytes from patients with rheumatoid arthritis. Apoptosis (2019) 24(11-12):892-904. Epub 2019/09/02. doi: 10.1007/s10495-019-01567-3. PubMed PMID: 31473844.

57. Raj Christian SD, Thirugnanasambantham K, Islam MIH, Sudalaimuthu MK, Sundaram S, Ashok G, et al. Identification of Expressed miRNAs in Human Rheumatoid Arthritis Using Computational Approach - Discovery of a New miR-7167 from Human. Microrna (2019) 8(2):147-54. Epub 2018/12/06. doi: 10.2174/2211536608666181204111438. PubMed PMID: 30514198. 
58. Smigielska-Czepiel K, van den Berg A, Jellema P, van der Lei RJ, Bijzet J, Kluiver J, et al. Comprehensive analysis of miRNA expression in $\mathrm{T}$ cell subsets of rheumatoid arthritis patients reveals defined signatures of naive and memory Tregs. Genes Immun (2014) 15(2):115-25. Epub 2014/01/10. doi: 10.1038/gene.2013.69. PubMed PMID: 24401767; PubMed Central PMCID: PMCPMC3959220.

59. Fulci V, Scappucci G, Sebastiani GD, Giannitti C, Franceschini D, Meloni F, et al. miR-223 is overexpressed in Tlymphocytes of patients affected by rheumatoid arthritis. Hum Immunol (2010) 71(2):206-11. Epub 2009/11/26. doi: 10.1016/j.humimm.2009.11.008. PubMed PMID: 19931339.

60. Rana AK, Li Y, Dang Q, Yang F. Monocytes in rheumatoid arthritis: Circulating precursors of macrophages and osteoclasts and, their heterogeneity and plasticity role in RA pathogenesis. International immunopharmacology (2018) 65:348-59. doi: 10.1016/j.intimp.2018.10.016. PubMed PMID: 30366278.

61. Lin J, Huo R, Xiao L, Zhu X, Xie J, Sun S, et al. A novel p53/microRNA-22/Cyr61 axis in synovial cells regulates inflammation in rheumatoid arthritis. Arthritis Rheumatol (2014) 66(1):49-59. Epub 2014/01/23. doi: 10.1002/art.38142. PubMed PMID: 24449575.

62. Tseng CC, Wu LY, Tsai WC, Ou TT, Wu CC, Sung WY, et al. Differential Expression Profiles of the Transcriptome and miRNA Interactome in Synovial Fibroblasts of Rheumatoid Arthritis Revealed by Next Generation Sequencing. Diagnostics (Basel) (2019) 9(3). Epub 2019/08/21. doi: 10.3390/diagnostics9030098. PubMed PMID: 31426562; PubMed Central PMCID: PMCPMC6787660.

63. Nakamachi Y, Kawano S, Takenokuchi M, Nishimura K, Sakai Y, Chin T, et al. MicroRNA-124a is a key regulator of proliferation and monocyte chemoattractant protein 1 secretion in fibroblast-like synoviocytes from patients with rheumatoid arthritis. Arthritis Rheum (2009) 60(5):1294-304. Epub 2009/05/01. doi: 10.1002/art.24475. PubMed PMID: 19404929.

64. Hong BK, You S, Yoo SA, Park D, Hwang D, Cho CS, et al. MicroRNA-143 and -145 modulate the phenotype of synovial fibroblasts in rheumatoid arthritis. Exp Mol Med (2017) 49(8):e363. Epub 2017/08/05. doi: 10.1038/emm.2017.108. PubMed PMID: 28775366; PubMed Central PMCID: PMCPMC5579506.

65. Yu S, Lu Y, Zong M, Tan Q, Fan L. Hypoxia-induced miR-191-C/EBPbeta signaling regulates cell proliferation and apoptosis of fibroblast-like synoviocytes from patients with rheumatoid arthritis. Arthritis Res Ther (2019) 21(1):78. Epub 2019/03/22. doi: 10.1186/s13075-019-1861-7. PubMed PMID: 30894209; PubMed Central PMCID: PMCPMC6425666.

66. Meng HY, Chen LQ, Chen LH. The inhibition by human MSCs-derived miRNA-124a overexpression exosomes in the proliferation and migration of rheumatoid arthritis-related fibroblast-like synoviocyte cell. BMC Musculoskelet Disord (2020) 21(1):150. Epub 2020/03/08. doi: 10.1186/s12891-020-3159-y. PubMed PMID: 32143603.

67. Wangyang Y, Yi L, Wang T, Feng Y, Liu G, Li D, et al. MiR-199a-3p inhibits proliferation and induces apoptosis in rheumatoid arthritis fibroblast-like synoviocytes via suppressing retinoblastoma 1. Biosci Rep (2018) 38(6). Epub 2018/10/26. doi: 10.1042/BSR20180982. PubMed PMID: 30352835; PubMed Central PMCID: PMCPMC6239273.

68. Cai Y, Jiang C, Zhu J, Xu K, Ren X, Xu L, et al. miR-449a inhibits cell proliferation, migration, and inflammation by regulating high-mobility group box protein 1 and forms a mutual inhibition loop with Yin Yang 1 in rheumatoid arthritis fibroblast-like synoviocytes. Arthritis Res Ther (2019) 21(1):134. Epub 2019/06/05. doi: 10.1186/s13075-019-1920-0. PubMed PMID: 31159863; PubMed Central PMCID: PMCPMC6547523.

69. Tolboom TC, Pieterman E, van der Laan WH, Toes RE, Huidekoper AL, Nelissen RG, et al. Invasive properties of fibroblast-like synoviocytes: correlation with growth characteristics and expression of MMP-1, MMP-3, and MMP-10. Ann Rheum Dis (2002) 61(11):975-80. doi: 10.1136/ard.61.11.975. PubMed PMID: 12379519; PubMed Central PMCID: PMC1753950.

70. Stanczyk J, Pedrioli DM, Brentano F, Sanchez-Pernaute O, Kolling C, Gay RE, et al. Altered expression of MicroRNA in synovial fibroblasts and synovial tissue in rheumatoid arthritis. Arthritis Rheum (2008) 58(4):1001-9. Epub 2008/04/03. doi: 10.1002/art.23386. PubMed PMID: 18383392.

71. Long L, Yu P, Liu Y, Wang S, Li R, Shi J, et al. Upregulated microRNA-155 expression in peripheral blood mononuclear cells and fibroblast-like synoviocytes in rheumatoid arthritis. Clin Dev Immunol (2013) 2013:296139. Epub 2013/10/24. doi: 10.1155/2013/296139. PubMed PMID: 24151514; PubMed Central PMCID: PMCPMC3789322.

72. Shi DL, Shi GR, Xie J, Du XZ, Yang H. MicroRNA-27a Inhibits Cell Migration and Invasion of Fibroblast-Like Synoviocytes by Targeting Follistatin-Like Protein 1 in Rheumatoid Arthritis. Mol Cells (2016) 39(8):611-8. Epub 2016/08/09. doi: 10.14348/molcells.2016.0103. PubMed PMID: 27498552; PubMed Central PMCID: PMCPMC4990753. 
73. de la Rica L, Urquiza JM, Gomez-Cabrero D, Islam AB, Lopez-Bigas N, Tegner J, et al. Identification of novel markers in rheumatoid arthritis through integrated analysis of DNA methylation and microRNA expression. J Autoimmun (2013) 41:6-16. Epub 2013/01/12. doi: 10.1016/j.jaut.2012.12.005. PubMed PMID: 23306098.

74. Okamoto K, Nakashima T, Shinohara M, Negishi-Koga T, Komatsu N, Terashima A, et al. Osteoimmunology: The Conceptual Framework Unifying the Immune and Skeletal Systems. Physiological reviews (2017) 97(4):1295-349. doi: 10.1152/physrev.00036.2016. PubMed PMID: 28814613.

75. Maeda Y, Farina NH, Matzelle MM, Fanning PJ, Lian JB, Gravallese EM. Synovium-Derived MicroRNAs Regulate Bone Pathways in Rheumatoid Arthritis. J Bone Miner Res (2017) 32(3):461-72. Epub 2016/10/30. doi: 10.1002/jbmr.3005. PubMed PMID: 27676131; PubMed Central PMCID: PMCPMC5340607.

76. Iwamoto N, Fukui S, Takatani A, Shimizu T, Umeda M, Nishino A, et al. Osteogenic differentiation of fibroblast-like synovial cells in rheumatoid arthritis is induced by microRNA-218 through a ROBO/Slit pathway. Arthritis Res Ther (2018) 20(1):189. Epub 2018/08/31. doi: 10.1186/s13075-018-1703-z. PubMed PMID: 30157923; PubMed Central PMCID: PMCPMC6116572.

77. Kurowska W, Kuca-Warnawin E, Radzikowska A, Jakubaszek M, Maslinska M, Kwiatkowska B, et al. Monocyte-related biomarkers of rheumatoid arthritis development in undifferentiated arthritis patients - a pilot study. Reumatologia (2018) 56(1):10-6. Epub 2018/04/25. doi: 10.5114/reum.2018.74742. PubMed PMID: 29686437; PubMed Central PMCID: PMCPMC5911652.

78. Ouboussad L, Hunt L, Hensor EMA, Nam JL, Barnes NA, Emery P, et al. Profiling microRNAs in individuals at risk of progression to rheumatoid arthritis. Arthritis Res Ther (2017) 19(1):288. Epub 2017/12/24. doi: 10.1186/s13075-017-1492-9. PubMed PMID: 29273071; PubMed Central PMCID: PMCPMC5741901.

79. Anaparti V, Smolik I, Meng X, Spicer V, Mookherjee N, El-Gabalawy H. Whole blood microRNA expression pattern differentiates patients with rheumatoid arthritis, their seropositive first-degree relatives, and healthy unrelated control subjects. Arthritis Res Ther (2017) 19(1):249. Epub 2017/11/12. doi: 10.1186/s13075-017-1459-x. PubMed PMID: 29126434; PubMed Central PMCID: PMCPMC5681796.

80. Yue J, Lau TCK, Griffith JF, Xu J, Xiao F, Shi L, et al. Circulating miR-99b-5p as a novel predictor of erosion progression on high-resolution peripheral quantitative computed tomography in early rheumatoid arthritis: A prospective cohort study. Int $J$ Rheum Dis (2019) 22(9):1724-33. Epub 2019/07/06. doi: 10.1111/1756-185X.13644. PubMed PMID: 31273939.

81. Evangelatos G, Fragoulis GE, Koulouri V, Lambrou GI. MicroRNAs in rheumatoid arthritis: From pathogenesis to clinical impact. Autoimmun Rev (2019) 18(11):102391. Epub 2019/09/15. doi: 10.1016/j.autrev.2019.102391. PubMed PMID: 31520804. 82. Mookherjee N, El-Gabalawy HS. High degree of correlation between whole blood and PBMC expression levels of miR-155 and miR-146a in healthy controls and rheumatoid arthritis patients. J Immunol Methods (2013) 400-401:106-10. Epub 2013/10/15. doi: 10.1016/j.jim.2013.10.001. PubMed PMID: 24120842.

83. Murata K, Furu M, Yoshitomi H, Ishikawa M, Shibuya H, Hashimoto M, et al. Comprehensive microRNA analysis identifies miR-24 and miR-125a-5p as plasma biomarkers for rheumatoid arthritis. PLoS One (2013) 8(7):e69118. Epub 2013/07/23. doi: 10.1371/journal.pone.0069118. PubMed PMID: 23874885; PubMed Central PMCID: PMCPMC3715465.

84. Ormseth MJ, Solus JF, Vickers KC, Oeser AM, Raggi P, Stein CM. Utility of Select Plasma MicroRNA for Disease and Cardiovascular Risk Assessment in Patients with Rheumatoid Arthritis. J Rheumatol (2015) 42(10):1746-51. Epub 2015/08/04. doi: 10.3899/jrheum.150232. PubMed PMID: 26233505; PubMed Central PMCID: PMCPMC4592411.

85. Wang W, Zhang Y, Zhu B, Duan T, Xu Q, Wang R, et al. Plasma microRNA expression profiles in Chinese patients with rheumatoid arthritis. Oncotarget (2015) 6(40):42557-68. Epub 2015/12/08. doi: 10.18632/oncotarget.6449. PubMed PMID: 26637811; PubMed Central PMCID: PMCPMC4767452.

86. Khalifa O, Pers YM, Ferreira R, Senechal A, Jorgensen C, Apparailly F, et al. X-Linked miRNAs Associated with Gender Differences in Rheumatoid Arthritis. Int J Mol Sci (2016) 17(11). Epub 2016/11/12. doi: 10.3390/ijms17111852. PubMed PMID: 27834806; PubMed Central PMCID: PMCPMC5133852.

87. Abou-Zeid A, Saad M, Soliman E. MicroRNA 146a expression in rheumatoid arthritis: association with tumor necrosis factor-alpha and disease activity. Genet Test Mol Biomarkers (2011) 15(11):807-12. Epub 2011/08/04. doi: 10.1089/gtmb.2011.0026. PubMed PMID: 21810022. 
88. Ormseth MJ, Solus JF, Sheng Q, Ye F, Song H, Wu Q, et al. The Endogenous Plasma Small RNAome of Rheumatoid Arthritis. ACR Open Rheumatol (2020) 2(2):97-105. Epub 2020/01/09. doi: 10.1002/acr2.11098. PubMed PMID: 31913579; PubMed Central PMCID: PMCPMC7011423.

89. Liu C, Pan A, Chen X, Tu J, Xia X, Sun L. MiR-5571-3p and miR-135b-5p, derived from analyses of microRNA profile sequencing, correlate with increased disease risk and activity of rheumatoid arthritis. Clin Rheumatol (2019) 38(6):1753-65. Epub 2019/02/02. doi: 10.1007/s10067-018-04417-w. PubMed PMID: 30707326.

90. Fernandez-Ruiz JC, Ramos-Remus C, Sanchez-Corona J, Castillo-Ortiz JD, Castaneda-Sanchez JJ, Bastian Y, et al. Analysis of miRNA expression in patients with rheumatoid arthritis during remission and relapse after a 5-year trial of tofacitinib treatment. International immunopharmacology (2018) 63:35-42. Epub 2018/08/04. doi: 10.1016/j.intimp.2018.07.028. PubMed PMID: 30075427.

91. Liu X, Ni S, Li C, Xu N, Chen W, Wu M, et al. Circulating microRNA-23b as a new biomarker for rheumatoid arthritis. Gene (2019) 712:143911. Epub 2019/06/10. doi: 10.1016/j.gene.2019.06.001. PubMed PMID: 31176730; PubMed Central PMCID: PMCPMC6724744.

92. Ormseth MJ, Solus JF, Sheng Q, Ye F, Wu Q, Guo Y, et al. Development and Validation of a MicroRNA Panel to Differentiate Between Patients with Rheumatoid Arthritis or Systemic Lupus Erythematosus and Controls. J Rheumatol (2020) 47(2):188-96. Epub 2019/05/17. doi: 10.3899/jrheum.181029. PubMed PMID: 31092710; PubMed Central PMCID: PMCPMC6856415.

93. Hong H, Yang H, Xia Y. Circulating miR-10a as Predictor of Therapy Response in Rheumatoid Arthritis Patients Treated with Methotrexate. Curr Pharm Biotechnol (2018) 19(1):79-86. Epub 2018/04/19. doi: 10.2174/1389201019666180417155140. PubMed PMID: 29667551.

94. Sode J, Krintel SB, Carlsen AL, Hetland ML, Johansen JS, Horslev-Petersen K, et al. Plasma MicroRNA Profiles in Patients with Early Rheumatoid Arthritis Responding to Adalimumab plus Methotrexate vs Methotrexate Alone: A Placebo-controlled Clinical Trial. J Rheumatol (2018) 45(1):53-61. Epub 2017/11/17. doi: 10.3899/jrheum.170266. PubMed PMID: 29142030. 95. Ciechomska M, Bonek K, Merdas M, Zarecki P, Swierkot J, Gluszko P, et al. Changes in MiRNA-5196 Expression as a Potential Biomarker of Anti-TNF-alpha Therapy in Rheumatoid Arthritis and Ankylosing Spondylitis Patients. Arch Immunol Ther Exp (Warsz) (2018) 66(5):389-97. Epub 2018/05/11. doi: 10.1007/s00005-018-0513-y. PubMed PMID: 29744553; PubMed Central PMCID: PMCPMC6154007.

96. Castro-Villegas C, Perez-Sanchez C, Escudero A, Filipescu I, Verdu M, Ruiz-Limon P, et al. Circulating miRNAs as potential biomarkers of therapy effectiveness in rheumatoid arthritis patients treated with anti-TNFalpha. Arthritis Res Ther (2015) 17:49. Epub 2015/04/11. doi: 10.1186/s13075-015-0555-z. PubMed PMID: 25860297; PubMed Central PMCID: PMCPMC4377058.

97. Liu Y, Han Y, Qu H, Fang J, Ye M, Yin W. Correlation of microRNA expression profile with clinical response to tumor necrosis factor inhibitor in treating rheumatoid arthritis patients: A prospective cohort study. J Clin Lab Anal (2019) 33(7):e22953. Epub 2019/06/28. doi: 10.1002/jcla.22953. PubMed PMID: 31245894; PubMed Central PMCID: PMCPMC6757134.

98. Duroux-Richard I, Pers YM, Fabre S, Ammari M, Baeten D, Cartron G, et al. Circulating miRNA-125b is a potential biomarker predicting response to rituximab in rheumatoid arthritis. Mediators Inflamm (2014) 2014:342524. Epub 2014/04/30. doi: 10.1155/2014/342524. PubMed PMID: 24778468; PubMed Central PMCID: PMCPMC3980876.

99. Perez-Sanchez C, Cecchi I, Barbarroja N, Patino-Trives AM, Luque-Tevar M, Perez-Sanchez L, et al. Early restoration of immune and vascular phenotypes in systemic lupus erythematosus and rheumatoid arthritis patients after B cell depletion. $J$ Cell Mol Med (2019) 23(9):6308-18. Epub 2019/07/28. doi: 10.1111/jcmm.14517. PubMed PMID: 31347786; PubMed Central PMCID: PMCPMC6714224.

100. Balzano F, Deiana M, Dei Giudici S, Oggiano A, Pasella S, Pinna S, et al. MicroRNA Expression Analysis of Centenarians and Rheumatoid Arthritis Patients Reveals a Common Expression Pattern. Int J Med Sci (2017) 14(7):622-8. Epub 2017/08/22. doi: 10.7150/ijms.18972. PubMed PMID: 28824293; PubMed Central PMCID: PMCPMC5562112.

101. Zhang Y, Wang H, Mao X, Guo Q, Li W, Wang X, et al. A Novel Circulating miRNA-Based Model Predicts the Response to Tripterysium Glycosides Tablets: Moving Toward Model-Based Precision Medicine in Rheumatoid Arthritis. Front Pharmacol (2018) 9:378. Epub 2018/06/09. doi: 10.3389/fphar.2018.00378. PubMed PMID: 29881347; PubMed Central PMCID:

PMCPMC5977984. 
102. Wang XY, Wang HL, Mao X, Li GY, Guo QY, Li WJ, et al. [Identification of biomarkers to response of Tripterygium Glycosides Tablets acting on rheumatoid arthritis by integrating transcriptional data mining and biomolecular network analysis]. Zhongguo Zhong Yao Za Zhi (2019) 44(16):3415-22. Epub 2019/10/12. doi: 10.19540/j.cnki.cjcmm.20181031.001. PubMed PMID: 31602903.

103. Mallinson DJ, Dunbar DR, Ridha S, Sutton ER, De la Rosa O, Dalemans W, et al. Identification of Potential Plasma microRNA Stratification Biomarkers for Response to Allogeneic Adipose-Derived Mesenchymal Stem Cells in Rheumatoid Arthritis. Stem Cells Transl Med (2017) 6(4):1202-6. Epub 2017/02/12. doi: 10.1002/sctm.16-0356. PubMed PMID: 28186687; PubMed Central PMCID: PMCPMC5442839.

104. Wang Y, Dai L, Wu H, Zhang ZR, Wang WY, Fu J, et al. Novel anti-inflammatory target of geniposide: Inhibiting Itgbeta1/Ras-Erk1/2 signal pathway via the miRNA-124a in rheumatoid arthritis synovial fibroblasts. International immunopharmacology (2018) 65:284-94. Epub 2018/10/21. doi: 10.1016/j.intimp.2018.09.049. PubMed PMID: 30342345.

105. Wu J, Fan W, Ma L, Geng X. miR-708-5p promotes fibroblast-like synoviocytes' cell apoptosis and ameliorates rheumatoid arthritis by the inhibition of Wnt3a/beta-catenin pathway. Drug Des Devel Ther (2018) 12:3439-47. Epub 2018/10/24. doi: 10.2147/DDDT.S177128. PubMed PMID: 30349197; PubMed Central PMCID: PMCPMC6186895.

106. Chen Z, Wang H, Xia Y, Yan F, Lu Y. Therapeutic Potential of Mesenchymal Cell-Derived miRNA-150-5p-Expressing Exosomes in Rheumatoid Arthritis Mediated by the Modulation of MMP14 and VEGF. J Immunol (2018) 201(8):2472-82. Epub 2018/09/19. doi: 10.4049/jimmunol.1800304. PubMed PMID: 30224512; PubMed Central PMCID: PMCPMC6176104.

107. Oka S, Furukawa H, Shimada K, Hashimoto A, Komiya A, Fukui N, et al. Plasma miRNA expression profiles in rheumatoid arthritis associated interstitial lung disease. BMC Musculoskelet Disord (2017) 18(1):21. Epub 2017/01/21. doi: 10.1186/s12891017-1389-4. PubMed PMID: 28103855; PubMed Central PMCID: PMCPMC5244611.

108. Li Z, Li Y, Li Q, Zhang Z, Jiang L, Li X. Role of miR-9-5p in preventing peripheral neuropathy in patients with rheumatoid arthritis by targeting REST/miR-132 pathway. In Vitro Cell Dev Biol Anim (2019) 55(1):52-61. Epub 2018/11/21. doi: 10.1007/s11626-018-0310-2. PubMed PMID: 30456455.

109. Cuppen BV, Rossato M, Fritsch-Stork RD, Concepcion AN, Schenk Y, Bijlsma JW, et al. Can baseline serum microRNAs predict response to TNF-alpha inhibitors in rheumatoid arthritis? Arthritis Res Ther (2016) 18:189. Epub 2016/08/26. doi: 10.1186/s13075-016-1085-z. PubMed PMID: 27558398; PubMed Central PMCID: PMCPMC4997731.

\section{Figure Legends:}




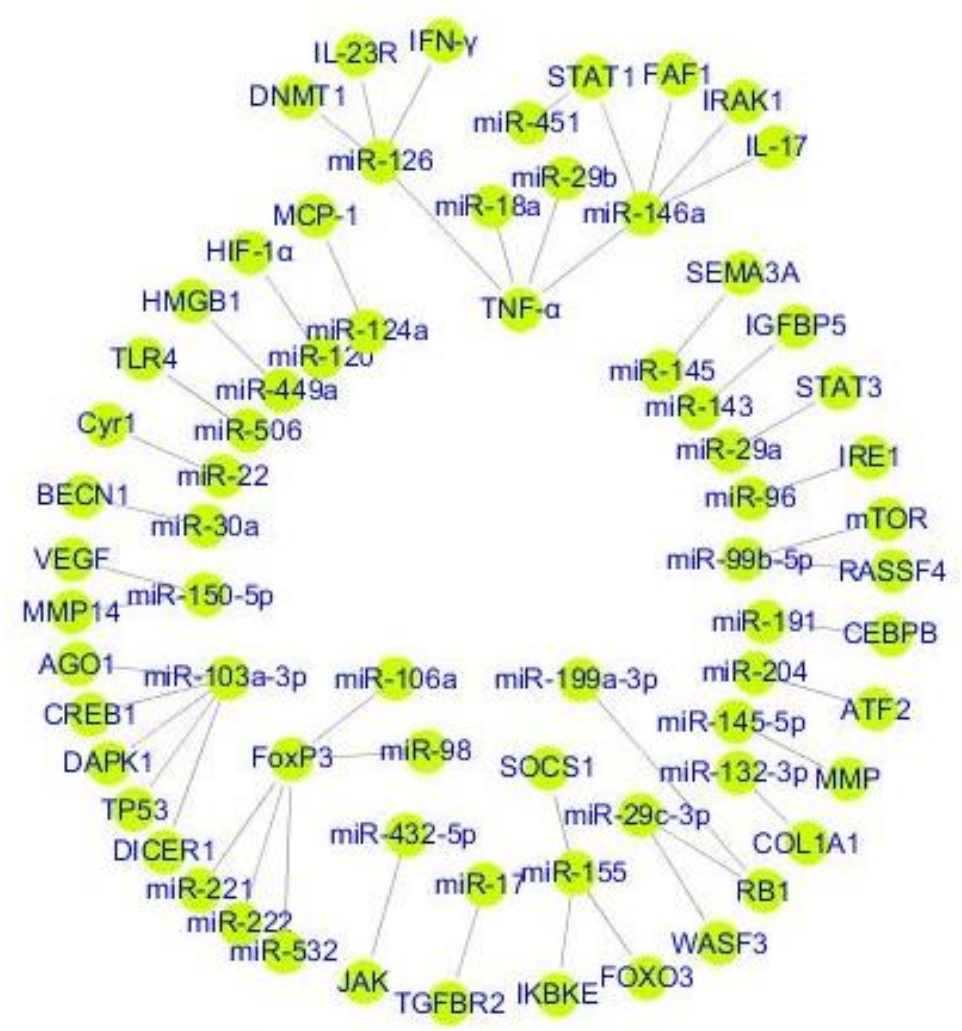

Figure 1. miRNA based regulatory network in rheumatoid arthritis. We extract all the regulatory network from the included studies and constructed the regulator network based on cytoscape. We can find numerous studies are focusing on TNF-a and cytokines while some other studies focusing on epigenetic regulation and inflammatory pathways. 